\title{
Correction to: ECML PKDD 2018 Workshops
}

\author{
Carlos Alzate (1) and Anna Monreale (1)
}

\section{Correction to:}

C. Alzate et al. (Eds.):

ECML PKDD 2018 Workshops, LNAI 11329,

https://doi.org/10.1007/978-3-030-13453-2

In the originally published version of the book, the editor "Irena Koprinska" was erroneously omitted from the editors' list. This has now been corrected. 